\title{
Surface topology specific metasurface holograms
}

\author{
James Burch, and Andrea Di Falco
}

ACS Photonics, Just Accepted Manuscript • DOI: 10.1021/acsphotonics.7b01449 • Publication Date (Web): 20 Jan 2018

Downloaded from http://pubs.acs.org on February 1, 2018

\section{Just Accepted}

"Just Accepted" manuscripts have been peer-reviewed and accepted for publication. They are posted online prior to technical editing, formatting for publication and author proofing. The American Chemical Society provides "Just Accepted" as a free service to the research community to expedite the dissemination of scientific material as soon as possible after acceptance. "Just Accepted" manuscripts appear in full in PDF format accompanied by an HTML abstract. "Just Accepted" manuscripts have been fully peer reviewed, but should not be considered the official version of record. They are accessible to all readers and citable by the Digital Object Identifier (DOI®). "Just Accepted" is an optional service offered to authors. Therefore, the "Just Accepted" Web site may not include all articles that will be published in the journal. After a manuscript is technically edited and formatted, it will be removed from the "Just Accepted" Web site and published as an ASAP article. Note that technical editing may introduce minor changes to the manuscript text and/or graphics which could affect content, and all legal disclaimers and ethical guidelines that apply to the journal pertain. ACS cannot be held responsible for errors or consequences arising from the use of information contained in these "Just Accepted" manuscripts. 


\title{
Surface topology specific metasurface holograms
}

\author{
James Burch* and Andrea Di Falco* \\ University of St Andrews, School of Physics and Astronomy, St Andrews, KY16 9SS, UK \\ E-mail: jb298@st-andrews.ac.uk; adf10@st-andrews.ac.uk \\ Phone: +44 1334461605
}

\begin{abstract}
Metasurface holograms are usually realized on flat surface topologies. Here we show that flexible holographic metasurfaces can be designed to form an image when applied to arbitrary substrate shapes. We demonstrate that for non-flat surfaces the phase contribution of the substrate determines the symmetry properties of the image. The sensitivity of the hologram to the specific surface topology can be enhanced by engineering the distribution of the phase elements in the metasurface area. The results of this work pave the way for practical applications in security printing technologies, in addition to surface topology and polarization sensors.
\end{abstract}

\section{Keywords}

Hologram, flexible, metasurface, multiplexing, conformable

Photonic Metasurfaces (MSs) are the ideal platform for the implementation of computer generated holograms. The associated subwavelength pixel resolution enables efficient design strategies $^{1-5}$ and the possibility to encode the information in different, independent degrees of freedom ${ }^{6}$ including polarization, ${ }^{7-11}$ wavelength, ${ }^{12,13}$ angle, ${ }^{14}$ and image dimensionality. ${ }^{15}$ Examples of applications of holographic MSs include displays, ${ }^{16}$ security ${ }^{8}$ and the manipulation and storage of information. ${ }^{17,18}$ 
The key enabling factor underpinning the success of MSs is the possibility to determine the phase and amplitude of the scattered light at subwavelength scales. The essentially twodimensional form factor of this class of devices is ideally exploited in flexible implementations. To date, flexible MSs have been successfully used for lab on fiber applications, cloaking, filtering and optically active three dimensional metamaterials. ${ }^{19-24}$

In the framework of holographic applications, flexibility has been exploited to create Flexible Holographic Metasurfaces (FHMSs) able to conform to not-flat substrates ${ }^{25}$ and to encode the holographic information in the degree of elastic stretching, e.g. to vary the focus of a lens ${ }^{26}$ and to switch between multiple images in the near field. ${ }^{27}$ FHMSs have also been explored theoretically to compensate for the spherical aberration of cylindrical lenses, and for carpet cloaking applications. ${ }^{28}$

Here we demonstrate experimentally that conformable FHMS can be designed to embed in their response the phase contribution of an uneven topology, for example inherited by a target substrate. In the following, we first derive the formalism to describe the interaction of light with arbitrarily shaped FHMS. In particular, we show how in non-flat FHMS the dependence of the holographic image from the handedness of the circular polarization of light is intrinsically linked to the shape of the substrate. We then demonstrate experimentally the encoding of information on both the FHMS and the shape of the substrates. Finally, we discuss a method to increase the sensitivity of the hologram to the exact shape of the FHMS.

\section{Generalized Gerchberg-Saxton algorithm}

To design the holograms we use the Gerchberg-Saxton algorithm (GSA). ${ }^{29}$ To deal with the general case of non-flat holographic MSs, we adopted the full Rayleigh-Sommerfeld formulation for light propagation, ${ }^{30}$ which links the complex light fields in the FHMS and the holographic image planes. 
An illustration of propagating light with the Rayleigh-Sommerfeld method is shown in Figure 1.

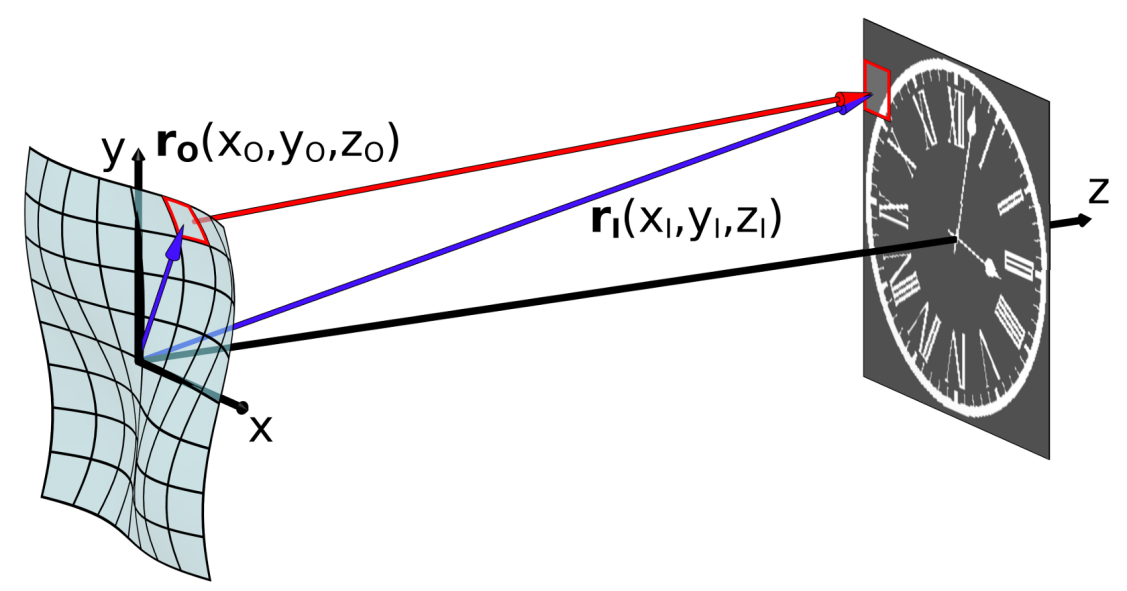

Figure 1: Illustration of light propagation using the Rayleigh-Sommerfeld equation from $\mathbf{r}_{O}\left(x_{O}, y_{O}, z_{O}\right)$ to $\mathbf{r}_{I}\left(x_{I}, y_{I}, z_{I}\right)$. The holographic image is projected from the MS onto the screen.

For most practical applications, the image is formed many wavelengths away from the FHMS plane, thus the near field contribution in the Rayleigh-Sommerfeld propagator can be neglected. In this case the Rayleigh-Sommerfeld equation simplifies into the Kirchoff equation:

$$
\begin{aligned}
U\left(x_{I}, y_{I}, z_{I}\right) & =-\iint U\left(x_{O}, y_{O}, z_{O}\right) \frac{2\left(z_{I}-z_{O}\right)}{\left|\mathbf{r}_{I}-\mathbf{r}_{O}\right|} \times i k \\
& \times \frac{\exp \left(i k\left|\mathbf{r}_{I}-\mathbf{r}_{O}\right|\right)}{4 \pi\left|\mathbf{r}_{I}-\mathbf{r}_{O}\right|} \times D\left(\mathbf{r}_{O}, \mathbf{r}_{I}\right) d x_{O} d y_{O}
\end{aligned}
$$

The complex light fields $U$ are calculated at the coordinates $\left(x_{j}, y_{j}, z_{j}\right)$, identified by the position vectors $\mathbf{r}_{j}$. The subscripts $j=O, I$ refer to the holographic MS object and holographic image planes respectively, and $k$ is the wavevector of light. The origin of the coordinate system is the center of a virtual, undistorted FHMS.

In eq 1 we included the directivity term $D\left(\mathbf{r}_{O}, \mathbf{r}_{I}\right)$, which depends on the angle between 
the local normal direction to the FHMS surface and the vector $\left(\mathbf{r}_{I}-\mathbf{r}_{O}\right)$, for each couple of points in the object and image planes. Crucially, the directivity incorporates the anisotropic scattering properties of the specific meta-atom of choice.

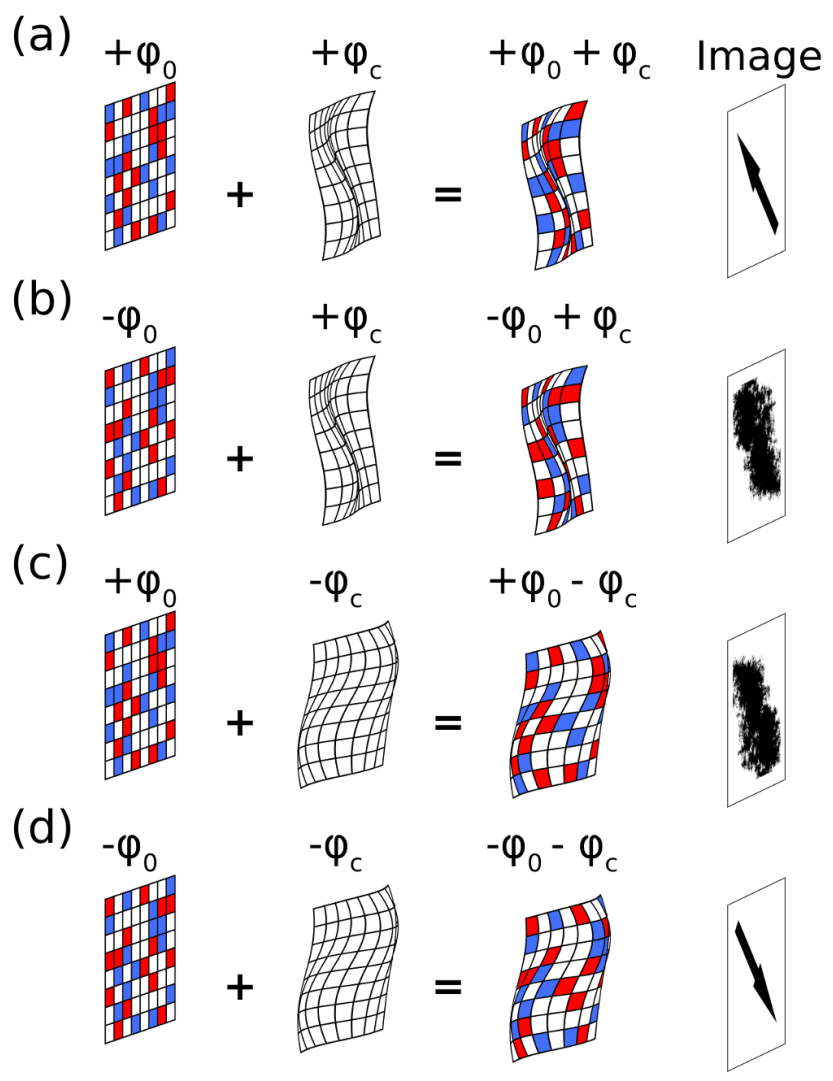

Figure 2: Simulated combinations of phases for a hologram with an arbitrary topology. Here we show a three level phase hologram for illustrative purposes. Pixels colored in red, blue, and white correspond to positive, negative, and no de-phasing respectively. $\phi_{0}\left(x_{O}, y_{O}, z_{O}\right)$ originates from the P-B phase required on a flat topology. $\phi_{c}\left(x_{O}, y_{O}, z_{O}\right)$ originates from the difference in the designed topology and the actual surface topology. (a) and (b) show the $\phi_{c}$ phase from an arbitrary topology. (c) and (d) show the $\phi_{c}$ phase from the same surface topology, but reflected in the $x y$ plane at $z=0$. (a) and (c) show right handed circularly polarized incident light. (b) and (c) show left handed circularly polarized incident light.

In flat FHMSs that rely on Pancharatnam-Berry (P-B) phases elements ${ }^{1,31}$ the information can be multiplexed in the handedness of circularly polarized light. For example, inverting the helicity of the light results in rotating the image 180 degrees around the zeroth order of the hologram, ${ }^{8}$ or into the formation of different images altogether. ${ }^{32,33}$ In the nonflat case the substrate topology introduces additional symmetry properties. From eq 1 it is 
possible to express the intensity of the holographic image as (for a fully detailed derivation see the SI):

$$
\begin{aligned}
I\left(x_{I}, y_{I}, z_{I}\right) & =\mid \Gamma\left(\mathbf{r}_{O}, \mathbf{r}_{I}\right) \iint \exp \left(i\left[\phi_{0}\left(x_{O}, y_{O}, z_{O}\right)+\phi_{c}\left(x_{O}, y_{O}, z_{O}\right)\right]\right) \\
& \times \exp \left(i k\left(z_{I}-z_{O}\right)\right) \times\left.\exp \left(i k \frac{\left(x_{I}-x_{O}\right)^{2}+\left(y_{I}-y_{O}\right)^{2}}{2\left(z_{I}-z_{O}\right)}\right) d x_{O} d y_{O}\right|^{2},
\end{aligned}
$$

where $I\left(x_{I}, y_{I}, z_{I}\right)$ is the intensity of light in the holographic image plane. Here, $\phi_{0}\left(x_{O}, y_{O}, z_{O}\right)$ is the phase of the P-B elements in the flat FHMS, and $\phi_{c}\left(x_{O}, y_{O}, z_{O}\right)$ is the phase contribution of the non-flat surface topology. The term

$$
\Gamma\left(\mathbf{r}_{O}, \mathbf{r}_{I}\right)=-\frac{2\left(z_{I}-z_{O}\right)}{\left|\mathbf{r}_{I}-\mathbf{r}_{O}\right|} \times \frac{i k}{4 \pi\left|\mathbf{r}_{I}-\mathbf{r}_{O}\right|}
$$

can be approximated to a constant amplitude value, if the angle between $r_{O}$ and $r_{I}$ is small, for which $r_{j} \simeq z_{j}$. It should also be noted that in deriving eq 2 the directivity term has been omitted, as it only provides for an amplitude correction. For flat FHMS $\phi_{c}=0$, and $\phi_{0}$ does not depend on the $z$ coordinate. In this case, changing the helicity of light maps $\phi_{0}\left(x_{O}, y_{O}, 0\right)$ into $-\phi_{0}\left(x_{O}, y_{O}, 0\right)$.

It is then possible to write

$$
I^{\prime}\left(x_{I}, y_{I}, z_{I}\right)=I\left(-x_{I},-y_{I}, z_{I}\right)
$$

which means that the holographic image is formed rotated 180 degrees around the $z$ axis, as expected. ${ }^{8,25}$ For a non-flat surface $\phi_{c}\left(x_{O}, y_{O}, z_{O}\right) \neq 0$ and eq 4 is not valid. However, if the FHMS is deformed so that $z_{O} \rightarrow-z_{O}, \phi_{c}\left(x_{O}, y_{O}, z_{O}\right)$ maps into $-\phi_{c}\left(x_{O}, y_{O}, z_{O}\right)$. In this case, eq 4 holds approximately true. A residual image distortion is caused by the relative 
de-phasing at $z_{I}$ for the two different configurations, which can be ignored for large $z_{I}$ (the full derivation is described in the SI). These cases are illustrated in Figure 2.

\section{Results and discussion}

To design the holograms we used the GSA with eq 1, for determined arbitrary substrate shapes. For the sake of simplicity, we considered the case of cylindrical substrates, without loss of generality. For more complex substrate shapes it is important to align the holographic metasurface to the topology of the substrate. This can be achieved by patterning standard alignment marks on both the sample, and the substrate, before using simple micro-positioning tools with the aid of an optical microscope. ${ }^{19}$ This technique allows for sub-micron resolution positioning, whilst being robust and repeatable. We constructed a FHMS that displays a holographic image when applied to a cylinder with radius $R=6 \mathrm{~mm}$, for circularly polarized light of wavelength $\lambda=630 \mathrm{~nm}$. The image was designed to be $30 \times 30 \mathrm{~mm}^{2}$ wide at a distance of $80 \mathrm{~mm}$ from the FHMS, with a resolution of $183 \times 183$ pixels. To avoid interference with the unconverted light, the image was offset $35 \mathrm{~mm}$ above the $z$ axis. For the practical implementation of the FHMS, we chose a three layer reflective type geometry, which ensures high diffraction efficiency. The curved topology does not affect the efficiency of the holograms, which is similar to that of typical three layers reflective type geometries. ${ }^{1,8,25}$ In this scheme, metallic nanorods are separated by a dielectric spacer from a reflective background plane, to act as P-B phase elements. Each nanorod imparts a de-phasing proportional to its orientation in the FHMS plane, with sign depending on the handedness of the polarization. ${ }^{1,8}$ This scheme is shown in Figure S2, along with a SEM image of a patterned FHMS. The nanorods have dimensions of $200 \times 75 \times 40 \mathrm{~nm}^{3}$, with a pitch of $300 \mathrm{~nm}$ between adjacent elements. In total we used $2501 \times 2501$ nanorods, covering a total area of $0.75 \times 0.75 \mathrm{~mm}^{2}$. The fabrication procedure followed a well tested approach. ${ }^{25}$ First we spin coated a polymeric sacrificial layer on a silicon substrate. We then spun a $2 \mu \mathrm{m}$ 
thick polymer (SU-8, Microchem) on top, to act as flexible substrate. Next we evaporated a $100 \mathrm{~nm}$ thick layer of gold to act as a backplane mirror, and after spinning a $90 \mathrm{~nm}$ thin layer of SU-8, we deposited a further $40 \mathrm{~nm}$ thick layer of gold for the nanorods. To pattern the top surface, we used standard top down e-beam lithography procedure, using a reactive ion etch step to remove the unmasked gold. Lastly, we dissolved the sacrificial layer to leave a free floating membrane, which could then be applied to any surface of choice. The directivity of the nanorods was calculated with the CST simulation package (see Figure S1 for the details).

The relationship between helicity and surface profile is displayed in Figure 3. A clear image is formed for left handed circularly polarized light on a concave cylindrical surface, with a radius of curvature of $6 \mathrm{~mm}$. A slightly distorted image, rotated 180 degrees around the $z$ axis, can be seen for right handed circularly polarized light on a convex cylindrical surface, again with a radius of curvature of $6 \mathrm{~mm}$. As expected in the cases of right handed circularly polarized light on a concave cylindrical surface and left handed circularly polarized light on a convex cylindrical surface, the holographic image is severely distorted.

The strong dependence of the image quality from a determined substrate shape could be harnessed for sensing and anti-counterfeiting applications. To measure quantitatively the sensitivity of the FHMS to deformations from the intended topologies we fabricated different samples, for different radii of curvature. Specifically, we designed the FHMS to form clear holographic images when applied to a flat surface, and to convex cylinders with radii of $4 \mathrm{~mm}$ and $6 \mathrm{~mm}$. The numerical and experimental results are in excellent agreement, as visible in Figure 4. A high fidelity image is only seen when the radius of curvature of the object matches that of the design. Furthermore, the greater the difference between the designed and the actual radius of curvature of the FHMS, the greater the distortion in the holographic image.

The distortion of the image can be quantified by a correlation coefficient, normalised to a value of 1 for identical images and -1 for perfectly complementary images. The correlation 

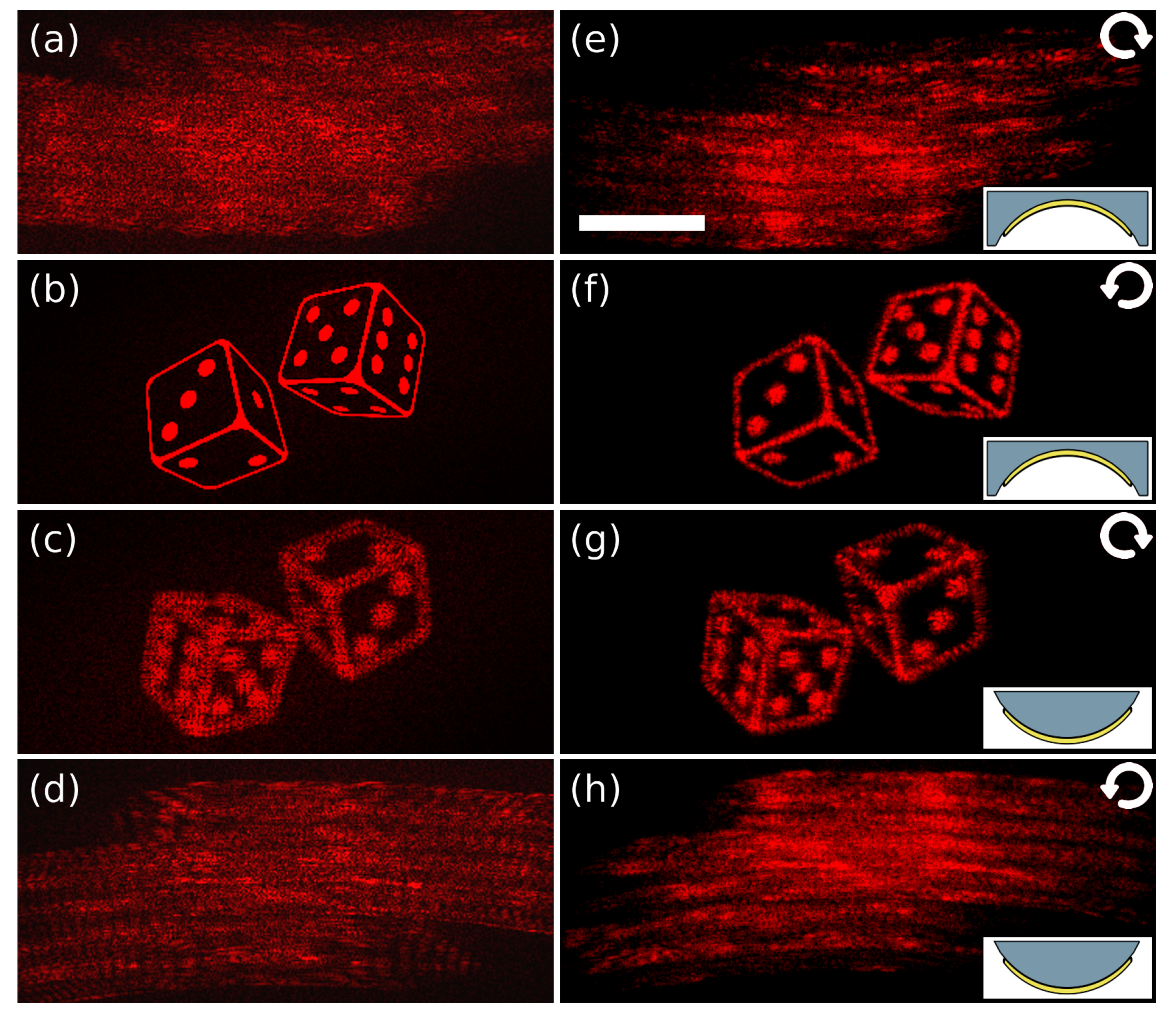

Figure 3: (a-d) display simulated holographic images, (e-h) display experimental holographic images. (a,e) and $(b, f)$ were taken on a concave substrate with a radius of curvature of $6 \mathrm{~mm}$. $(\mathrm{c}, \mathrm{g})$ and $(\mathrm{d}, \mathrm{h})$ were taken on a convex substrate with the same radius of curvature. $(\mathrm{a}, \mathrm{e})$ and $(\mathrm{c}, \mathrm{g})$ were taken with right handed circularly polarized incident light. $(\mathrm{b}, \mathrm{f})$ and $(\mathrm{d}, \mathrm{h})$ were taken with left handed circularly polarized incident light. The left handed circularly polarized images are naturally rotated 180 degrees about the $z$ axis in the $x y$ plane. The scale bar is equivalent to $10 \mathrm{~mm}$.
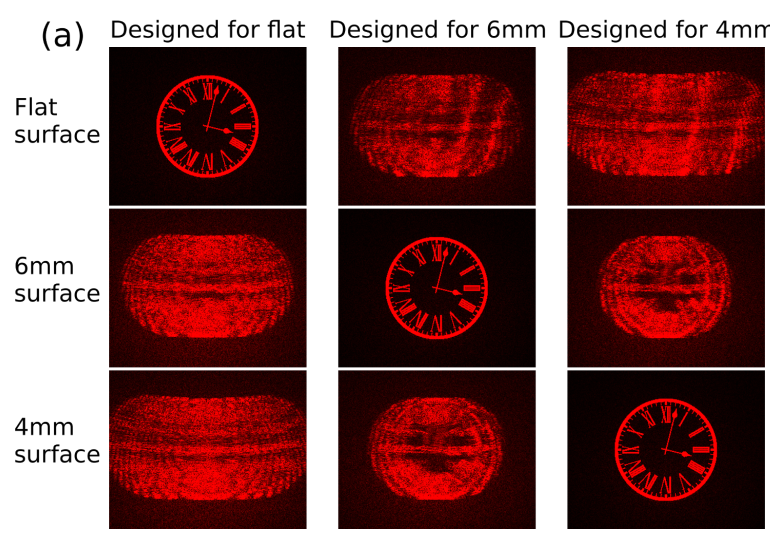

(b)

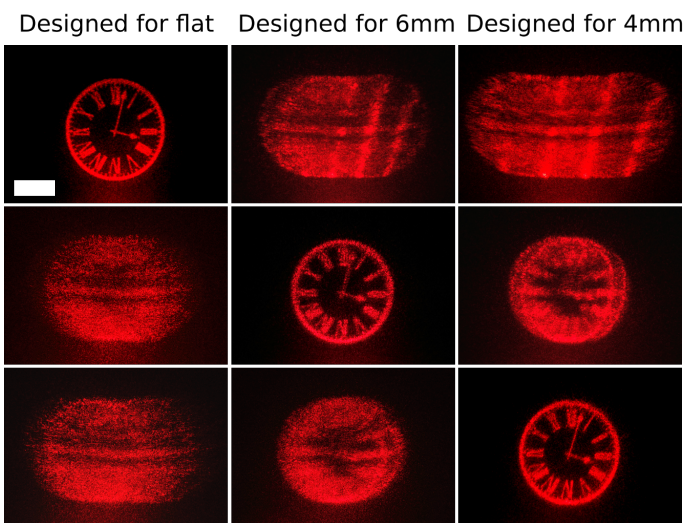

Figure 4: (a) Simulated results displaying holograms designed for different convex cylindrical radii of curvature placed on different convex cylindrical radii of curvature. (b) Experimental results displaying holograms designed for 3 different cylindrical radii of curvature conformed to 3 glass cylinders with different radii of curvature. The scale bar is equivalent to $10 \mathrm{~mm}$. 

substrate topologies

\begin{tabular}{c|ccc} 
& \multicolumn{3}{|c}{ Design } \\
Applied & $\mathrm{R}=\infty$ & $\mathrm{R}=6 \mathrm{~mm}$ & $\mathrm{R}=4 \mathrm{~mm}$ \\
\hline $\mathrm{R}=\infty$ & 0.9635 & 0.3439 & 0.2975 \\
$\mathrm{R}=6 \mathrm{~mm}$ & 0.3570 & 0.9640 & 0.4518 \\
$\mathrm{R}=4 \mathrm{~mm}$ & 0.3003 & 0.4458 & 0.9652
\end{tabular}

The selectivity depends from the difference between the designed topology and the actual shape of the substrate. This effect can be enhanced if the information is encoded in the regions of the FHMS that have the highest gradient relative to the designed topology. Clearly, reducing the effective area of the phase pattern has the detrimental effects of decreasing both the intensity of the holographic image, and the width of its spectral content. The sensitivity also depends from the size of the holographic image, since smaller features will be affected more by the same amount of deformation. For the cylindrical case, we designed FHMS with an increasingly large gap $\eta$ (expressed as fraction of the side of the FHMS), in the central section of the phase pattern. The results are summarized in Figure 5. In panel a) we illustrate an example of phase patterns of FHMSs with varying gap width, designed to create the same target image of Figure 4, for a cylinder of $R=6 \mathrm{~mm}$. Panel b) shows the correlation coefficient between the resulting projected images, and the target image (Figure S3 shows a collection of the projected images). As expected, wider phase gaps lead to an enhanced selectivity, at the cost of a slight reduction in intensity and spectral resolution.

In conclusion, we have demonstrated that conformable FHMS can be designed to form a holographic image that depends on the specific surface topology. We demonstrated theoretically and experimentally that the symmetry properties of the formed images depend from both the helicity of the polarization and the phase contribution of the substrate. The specificity of the hologram can be enhanced by engineering the phase distribution in the FHMS, at the cost of a small loss of holographic image quality. We anticipate that these results will find applications in surface topology sensing and anti-counterfeiting. 
(a)

$\eta=0.00$

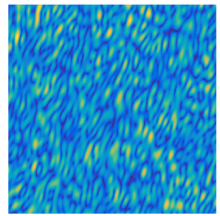

$\eta=0.30$

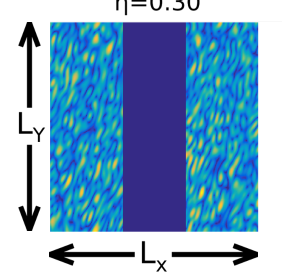

$\eta=0.15$

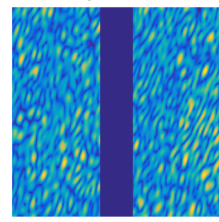

$\eta=0.45$

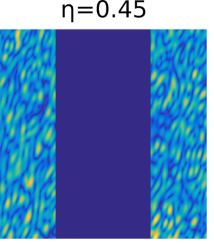

(b)

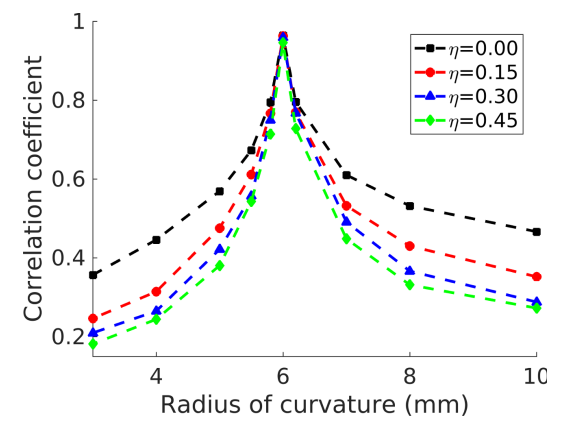

Figure 5: (a) Sketch of the spatial phase distributions encoded onto the FHMS for different $\eta$ values. (b) Correlation coefficient vs radius of curvature for the phase profile for different phase gaps.

\section{Acknowledgement}

The authors thank Xiang-kun Kong of Nanjing University of Aeronautics and Astronautics for the CST simulations of the directivity. J.B. and A.D.F acknowledge support from EPSRC (grants No EP/M508214/1, EP/L017008/1). The research data supporting this publication can be accessed at http://dx.doi.org/10.17630/583a258e-32cb-4a6b-826c-ab7d3b4429e2.

\section{Supporting Information Available}

- SupplementaryInformation.pdf: a pdf containing additional information.

This material is available free of charge via the Internet at http://pubs.acs.org/.

\section{References}

(1) Zheng, G.; Mühlenbernd, H.; Kenney, M.; Li, G.; Zentgraf, T.; Zhang, S. Metasurface holograms reaching 80\% efficiency. Nature Nanotechnology 2015, 10, 308-312.

(2) Arbabi, A.; Horie, Y.; Ball, A.; Bagheri, M.; Faraon, A. Subwavelength-thick lenses with 
high numerical apertures and large efficiency based on high-contrast transmitarrays. Nature Communications 2015, 6, 7069.

(3) Arbabi, A.; Horie, Y.; Bagheri, M.; Faraon, A. Dielectric metasurfaces for complete control of phase and polarization with subwavelength spatial resolution and high transmission. Nature Nanotechnology 2015, 10, 937-943.

(4) Wei, Z.; Cao, Y.; Su, X.; Gong, Z.; Long, Y.; Li, H. Highly efficient beam steering with a transparent metasurface. Optics Express 2013, 21, 10739-10745.

(5) Wang, L.; Kruk, S.; Tang, H.; Li, T.; Kravchenko, I.; Neshev, D. N.; Kivshar, Y. S. Grayscale transparent metasurface holograms. Optica 2016, 3, 1504-1505.

(6) Yu, N.; Capasso, F. Flat optics with designer metasurfaces. Nature Materials 2014, 13, 139-150.

(7) Liu, H.-C.; Yang, B.; Guo, Q.; Shi, J.; Guan, C.; Zheng, G.; Mühlenbernd, H.; Li, G.; Zentgraf, T.; Zhang, S. Single-pixel computational ghost imaging with helicitydependent metasurface hologram. Science Advances 2017, 3, e1701477.

(8) Wen, D.; Yue, F.; Li, G.; Zheng, G.; Chan, K.; Chen, S.; Chen, M.; Li, K. F.; Wong, P. W. H.; Cheah, K. W.; Pun, E. Y. B.; Zhang, S.; Chen, X. Helicity multiplexed broadband metasurface holograms. Nature Communications 2015, 6, 8241.

(9) Montelongo, Y.; Tenorio-Pearl, J.; Milne, W.; Wilkinson, T. Polarization switchable diffraction based on subwavelength plasmonic nanoantennas. Nano Letters 2013, 14, $294-298$.

(10) Ye, W.; Zeuner, F.; Li, X.; Reineke, B.; He, S.; Qiu, C.-W.; Liu, J.; Wang, Y.; Zhang, S.; Zentgraf, T. Spin and wavelength multiplexed nonlinear metasurface holography. Nature Communications 2016, 7, 11930. 
(11) Khorasaninejad, M.; Ambrosio, A.; Kanhaiya, P.; Capasso, F. Broadband and chiral binary dielectric meta-holograms. Science Advances 2016, 2, e1501258.

(12) Montelongo, Y.; Tenorio-Pearl, J. O.; Williams, C.; Zhang, S.; Milne, W. I.; Wilkinson, T. D. Plasmonic nanoparticle scattering for color holograms. Proceedings of the National Academy of Sciences 2014, 111, 12679-12683.

(13) Li, X.; Chen, L.; Li, Y.; Zhang, X.; Pu, M.; Zhao, Z.; Ma, X.; Wang, Y.; Hong, M.; Luo, X. Multicolor 3D meta-holography by broadband plasmonic modulation. Science Advances 2016, 2, e1601102.

(14) Kamali, S. M.; Arbabi, E.; Arbabi, A.; Horie, Y.; Faraji-Dana, M.; Faraon, A. Anglemultiplexed metasurfaces: encoding independent wavefronts in a single metasurface under different illumination angles. Phys Rev X 2017, 7, 041056.

(15) Huang, L.; Mühlenbernd, H.; Li, X.; Song, X.; Bai, B.; Wang, Y.; Zentgraf, T. Broadband hybrid holographic multiplexing with geometric metasurfaces. Advanced Materials 2015, 27, 6444-6449.

(16) Huang, L.; Chen, X.; Mühlenbernd, H.; Zhang, H.; Chen, S.; Bai, B.; Tan, Q.; Jin, G.; Cheah, K.-W.; Qiu, C.-W.; Li, J.; Zentgraf, T.; Zhang, S. Three-dimensional optical holography using a plasmonic metasurface. Nature Communications 2013, 4, 2808.

(17) Nobukawa, T.; Nomura, T. Multilayer recording holographic data storage using a varifocal lens generated with a kinoform. Optics Letters 2015, 40, 5419-5422.

(18) Shimada, K.-i.; Ide, T.; Shimano, T.; Anderson, K.; Curtis, K. New optical architecture for holographic data storage system compatible with Blu-ray Disc system. Optical Engineering 2014, 53, 025102-025102.

(19) Reader-Harris, P.; Di Falco, A. Nanoplasmonic filters for hollow core photonic crystal fibers. ACS Photonics 2014, 1, 985-989. 
(20) Reader-Harris, P.; Ricciardi, A.; Krauss, T.; Di Falco, A. Optical guided mode resonance filter on a flexible substrate. Optics Express 2013, 21, 1002-1007.

(21) Di Falco, A.; Ploschner, M.; Krauss, T. Flexible metamaterials at visible wavelengths. New Journal of Physics 2010, 12, 113006.

(22) Yang, S.; Liu, P.; Yang, M.; Wang, Q.; Song, J.; Dong, L. From flexible and stretchable meta-atom to metamaterial: A wearable microwave meta-skin with tunable frequency selective and cloaking effects. Scientific Reports 2016, 6, 21921.

(23) Walia, S.; Shah, C.; Gutruf, P.; Nili, H.; Chowdhury, D. R.; Withayachumnankul, W.; Bhaskaran, M.; Sriram, S. Flexible metasurfaces and metamaterials: A review of materials and fabrication processes at micro-and nano-scales. Applied Physics Reviews 2015, 2, 011303.

(24) Di Falco, A.; Zhao, Y.; Alú, A. Optical metasurfaces with robust angular response on flexible substrates. Applied Physics Letters 2011, 99, 163110.

(25) Burch, J.; Wen, D.; Xianzhong, C.; Di Falco, A. Conformable Holographic Metasurfaces. Scientific Reports 2017, 7, 4520.

(26) Ee, H.-S.; Agarwal, R. Tunable metasurface and flat optical zoom lens on a stretchable substrate. Nano Letters 2016, 16, 2818-2823.

(27) Malek, S. C.; Ee, H.-S.; Agarwal, R. Strain multiplexed metasurface holograms on a stretchable substrate. Nano Letters 2017, 17, 36413645.

(28) Cheng, J.; Jafar-Zanjani, S.; Mosallaei, H. All-dielectric ultrathin conformal metasurfaces: lensing and cloaking applications at $532 \mathrm{~nm}$ wavelength. Scientific Reports $\mathbf{2 0 1 6}$, 6,38440 .

(29) Gerchberg, R. A practical algorithm for the determination of phase from image and diffraction plane pictures. Optik 1972, 35, 237. 
(30) Veerman, J. A.; Rusch, J. J.; Urbach, H. P. Calculation of the Rayleigh-Sommerfeld diffraction integral by exact integration of the fast oscillating factor. JOSA A $\mathbf{2 0 0 5}$, 22, 636-646.

(31) Huang, Y.-W.; Chen, W. T.; Tsai, W.-Y.; Wu, P. C.; Wang, C.-M.; Sun, G.; Tsai, D. P. Aluminum plasmonic multicolor meta-hologram. Nano Letters 2015, 15, 3122-3127.

(32) Mueller, B.; Rubin, N.; Devlin, R.; Groever, B.; Capasso, F. Metasurface Polarization Optics: Independent Phase Control of Arbitrary Orthogonal States of Polarization. Physical Review Letters 2017, 118, 113901.

(33) Wen, D.; Chen, S.; Yue, F.; Chan, K.; Chen, M.; Ardron, M.; Li, K. F.; Wong, P. W. H.; Cheah, K. W.; Pun, E. Y. B.; Li, G.; Zhang, S.; Chen, X. Metasurface Device with Helicity-Dependent Functionality. Advanced Optical Materials 2016, 4, 321-327. 


\section{For Table of Contents Use Only}

Title: Surface topology specific metasurface holograms.

Authors: James Burch, Andrea Di Falco

On the left side of this figure we display an SEM image of the hologram, in addition to a photograph of the hologram conformed to a glass rod. On the right side the figure is comprised of two holographic images.

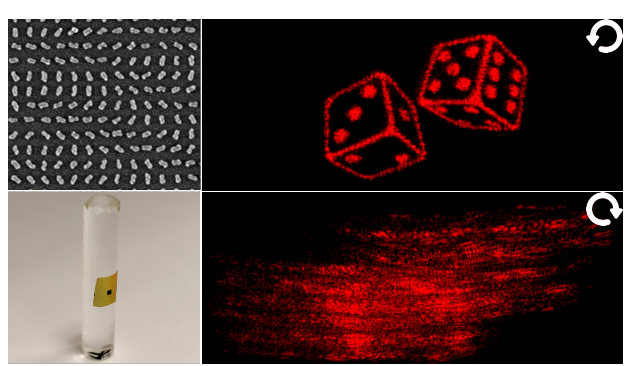

